\title{
PARTIAL INVERSION FOR LINEAR SYSTEMS AND PARTIAL CLOSURE OF INDEPENDENCE GRAPHS
}

\author{
N. WERMUTH ${ }^{1}$, M. WIEDENBECK ${ }^{2}$ and D. R. COX ${ }^{3}$ \\ ${ }^{1}$ Mathematical Statistics, Chalmers/Göteborgs Universitet, 41296 Göteborg, Sweden. \\ email: wermuth@math.chalmers.se \\ ${ }^{2}$ Center of Survey Research, B2, 1, 68159 Mannheim, Germany. \\ email: wiedenbeck@zuma-mannheim.de \\ ${ }^{3}$ Nuffield College, Oxford, OX1 1NF, UK. \\ email: david.cox@nuffield.oxford.ac.uk
}

\begin{abstract}
.
We introduce and study a calculus for real-valued square matrices, called partial inversion, and an associated calculus for binary square matrices. The first, applied to systems of recursive linear equations, generates new sets of parameters for different types of statistical joint response models. The corresponding generating graphs are directed and acyclic. The second calculus, applied to matrix representations of independence graphs, gives chain graphs induced by such a generating graph. Chain graphs are more complex independence graphs associated with recursive joint response models. Missing edges in independence graphs coincide with structurally zero parameters in linear systems. A wide range of consequences of an assumed independence structure can be derived by partial closure, but computationally efficient algorithms still need to be developed for applications to very large graphs.
\end{abstract}

AMS subject classification (2000): 00A71, 15A09, 15A23, 05C99, 62H99, 62J05.

Key words: Cholesky factorization, directed acyclic graphs, graphical chain model, Gauss-Doolittle method, induced independence graphs, successive orthogonalization, sweep operator, triangular systems.

\section{Introduction.}

Methods for solving linear equations, or equivalently for matrix inversion, were known in China more than 2000 years ago [40, 24]. In surveying such methods have been introduced under the name of Gauss-Jordan elimination [23] in German, of Cholesky-factorization [6] in French, and of the Gauss-Doolittle method [16] in American geodesic literature. In mathematics and statistics such methods for symmetric matrices have been called successive orthogonal-

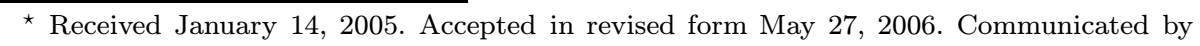
Iain Duff. 
ization $[22,36]$ and the sweep operator $[5,13]$. Aspects of matrix decomposition and computational efficiency have been studied in numerical analysis and computer science, see e.g. [18, 39, 21]. We introduce a calculus for partial inversion of real-valued matrices, derive its properties and relate it to different types of block-triangular decompositions of positive definite matrices.

Such matrix decompositions relate closely in a statistical context to linear graphical chain models. With graphical chain models [10, 17, 27, 42] one can formulate relations among many random variables of arbitrary distributional form, such that simplifying structure results from conditional independencies and is captured in graphs. In independence graphs nodes represent random variables and missing edges indicate sets of parameters constrained to take value zero and to capture independence. In such systems, there is typically a direction of dependence between some but not all pairs of nodes.

These multivariate statistical models combine three essential concepts which have been developed independently at the beginning of the 20th century. The geneticist Wright [46] used directed graphs to formulate hypotheses about how linear relations in his data could have been generated. The probabilist Markov [31] introduced the notion of conditional independence to represent seemingly complex structures by a sequence of univariate dependencies, and the physicist Gibbs [20] characterized the higher density of a substance by an undirected graph, in which nodes have a larger number of nearest neighbors. Many properties and estimation algorithms for different subclasses of graphical models have been established in the statistical literature in the last 30 years, but these have so far not been connected to properties of matrix operators.

Key questions in statistical modeling are: how is the strength and direction of dependence between two random variables changed when their set of conditioning variables is modified, and in which situations are both properties preserved. These questions concern the parameters of a model which are free to vary, often within some range of non-vanishing dependence. Answers are essential for comparing results of different empirical studies on the same set of core variables. In two studies of even the same set of variables, different sequences for the variables may be used for analysis or only a partial ordering be given, when some variables are to be considered as joint responses. Or, it may be that some variables are omitted, i.e. marginalized over, or a sub-population is studied for which some levels of other variables are held fixed, i.e. are conditioned on.

Closely related are enquiries into change and preservation of independence constraints specified by a given graph. For this, we introduce a calculus for finding structural zeros after partial inversion, derive its properties and apply it to graphical chain models. This calculus operates on binary matrices. Many preserved independencies typically simplify statistical analysis even when the interpretation of the constraints and of the unconstrained parameters is changed.

Needed for the new matrix results are a minor modification of the sweep operator, so that it becomes applicable to real-valued square matrices instead of only to symmetric matrices, and a minor modification of adjacency matrices, the binary matrix representations traditional in the graph theoretic literature, so 
that matrix products of the new binary matrices, called edge matrices, become analogous to the real-valued matrix products in partial inversion.

The plan of the paper is to introduce partial inversion in Section 2. In Section 3, partial inversion is applied to symmetric matrices and related to the statistical concept of linear least squares regression coefficients, to conditional covariance matrices and to inverse marginal covariance matrices. Different properties of these types of parameters motivate the use of independence graphs with several types of edge. In Section 4, the discussion is extended to parameter matrices of linear chain graph models induced after partial inversion and in Section 5 to chain graphs induced after partial closure of the generating graph. In a short final section we point to some open problems.

\section{Partial inversion and its properties.}

\subsection{Definition and basic properties.}

Let $N=\left\{1, \ldots, d_{N}\right\}$ be the index set of rows and corresponding columns of a square matrix $M$, whose principal submatrices are all invertible, i.e. for which the inverse of $M_{a a}=[M]_{a, a}$ exists for every nonempty subset $a$ of $N$, and is denoted by $M_{a a}^{-1}$. Let further $N$ be split into two arbitrary components $a$ and $b$, so that, if necessary after permuting rows and columns, we get $N=(a, b)$. For two real valued vectors $x$ and $y$ split accordingly, we are to introduce below in Equation (2.2) an operation on $M$, to be called partial inversion and denoted by $\operatorname{inv}_{a}$, such that for

$$
M\left(\begin{array}{l}
x_{a} \\
x_{b}
\end{array}\right)=\left(\begin{array}{l}
y_{a} \\
y_{b}
\end{array}\right)
$$

the linear relation after partial inversion is

$$
\operatorname{inv}_{a} M\left(\begin{array}{c}
y_{a} \\
x_{b}
\end{array}\right)=\left(\begin{array}{c}
x_{a} \\
y_{b}
\end{array}\right)
$$

For this we write $M$ and its inverse $M^{-1}$ in partitioned form as

$$
M=\left(\begin{array}{cc}
M_{a a} & M_{a b} \\
M_{b a} & M_{b b}
\end{array}\right), M^{-1}=\left(\begin{array}{cc}
M^{a a} & M^{a b} \\
M^{b a} & M^{b b}
\end{array}\right) .
$$

Partial inversion of $M$ on subset $a$ of $N$, and a convenient notation, are then defined by

$$
\operatorname{inv}_{a} M=\left(\begin{array}{rr}
M_{a a}^{-1} & -M_{a a}^{-1} M_{a b} \\
M_{b a} M_{a a}^{-1} & M_{b b}-M_{b a} M_{a a}^{-1} M_{a b}
\end{array}\right)=\left(\begin{array}{cr}
M_{a a}^{-1} & -M_{a-b} \\
M_{b \rightarrow a} & M_{b b . a}
\end{array}\right) .
$$

The notation $M_{a \leftarrow b}$ reminds us that the matrix $M_{a b}$ is multiplied from the left by $M_{a a}^{-1}$ and $M_{b b . a}$ is our notation for what is often called a Schur matrix. That the operator defined in Equation (2.2) is of the desired form in Equation (2.1), is verified by writing the component for $a$ from $M x=y$ as

$$
y_{a}=M_{a a} x_{a}+M_{a b} x_{b}
$$

and substituting it on the left-hand side of Equation (2.1). 
One use of partial inversion is to decompose matrix inversion into steps of the same kind, which lead to the inverse of $M$ if applied in sequence to each element of $N$. In the following, we study the properties of this operator, derive several recursion relations from it and show some of its applications to linear statistical models, and to matrix representations of graphs. Some of the results hold under weaker assumptions, but our main applications concern positive definite matrices and unit triangular matrices, i.e. triangular matrices having ones along the diagonal, for both of which all principal submatrices are invertible.

LEMMA 2.1. Basic properties of partial inversion.

(i) Partial inversion on component a is undone by reapplying it to a:

$$
\operatorname{inv}_{a} \operatorname{inv}_{a} M=M ;
$$

(ii) the matrix $M$ partially inverted on a is the inverse of $M$ after partial inversion on the remaining components $b$ :

$$
\operatorname{inv}_{a} M=\left(\operatorname{inv}_{b} M\right)^{-1}
$$

(iii) partial inversion on component a of $M$ followed by partial inversion on the remaining components $b$ gives the inverse of $M$ :

$$
\operatorname{inv}_{b} \operatorname{inv}_{a} M=M^{-1}
$$

(iv) the order of partial inversion on components a and $b$ can be interchanged:

$$
\operatorname{inv}_{b} \operatorname{inv}_{a} M=\operatorname{inv}_{a} \operatorname{inv}_{b} M
$$

( $v$ ) the matrix $M$ partially inverted on a coincides with its inverse $M^{-1}$ partially inverted on the remaining components $b$ :

$$
\operatorname{inv}_{a} M=\operatorname{inv}_{b} M^{-1}
$$

(vi) inversion and partial inversion can be interchanged:

$$
\left(\operatorname{inv}_{b} M\right)^{-1}=\operatorname{inv}_{b} M^{-1} .
$$

Proof. Properties $(i),(i i i)$ and $(i v)$ are direct from Equation (2.1), property $(v)$ results with property $(i)$ applied to partial inversion on $b$ in $(i i i)$, and $(v i)$ is direct from $(i i)$ and $(v)$.

For property $(i i)$ equality results from Equation (2.1) and

$$
\operatorname{inv}_{b} M\left(\begin{array}{c}
x_{a} \\
y_{b}
\end{array}\right)=\left(\begin{array}{c}
y_{a} \\
x_{b}
\end{array}\right), \quad\left(\operatorname{inv}_{b} M\right)^{-1}\left(\begin{array}{c}
y_{a} \\
x_{b}
\end{array}\right)=\left(\begin{array}{c}
x_{a} \\
y_{b}
\end{array}\right)
$$

and the proof is complete.

It is, however, instructive to prove the basic properties of the operator also directly by matrix calculations. Then, case $(i)$ results by applying partial inversion to component $a$ of the matrix $\operatorname{inv}_{a} M$ in Equation (2.2). Direct computation 
gives property $(i i)$, with

$$
\left(\begin{array}{cc}
M_{a a}^{-1} & -M_{a \vdash b} \\
M_{b \rightarrow a} & M_{b b . a}
\end{array}\right)\left(\begin{array}{cc}
M_{a a . b} & M_{a \rightarrow b} \\
-M_{b \vdash a} & M_{b b}^{-1}
\end{array}\right)=\left(\begin{array}{cc}
I_{a a} & 0_{a b} \\
0_{b a} & I_{b b}
\end{array}\right) .
$$

Partial inversion on component $b$ applied to $\operatorname{inv}_{a} M$ in Equation (2.2) can be written as

$$
\operatorname{inv}_{b} \operatorname{inv}_{a} M=\operatorname{inv}_{N} M=\left(\begin{array}{rr}
M_{a a}^{-1}+M_{a-b} M_{b b . a}^{-1} M_{b \rightarrow a} & -M_{a-b} M_{b b . a}^{-1} \\
-M_{b b . a}^{-1} M_{b \rightarrow a} & M_{b b . a}^{-1}
\end{array}\right)
$$

and

$$
\left(\begin{array}{ll}
M_{a a} & M_{a b}
\end{array}\right) \operatorname{inv}_{N} M=\left(\begin{array}{ll}
I_{a a} & 0_{a b}
\end{array}\right), \quad\left(\begin{array}{ll}
-M_{b b . a}^{-1} M_{b \rightarrow a} & M_{b b . a}^{-1}
\end{array}\right) M=\left(\begin{array}{ll}
0_{b a} & I_{b b}
\end{array}\right)
$$

proving that $M^{-1}$ has the form of the matrix in Equation (2.3), so that property $(i i i)$ holds. The exchangeability in case $(i v)$ follows from case $(i i i)$, where $a$, as well as $b$ are arbitrary, disjoint and nonempty subsets of $N$.

\subsection{Directly related results.}

Partial inversion in Equation (2.2) generalizes the sweep operator for symmetric matrices of Beaton [5] for which Dempster [13, 14] has shown that it has properties $(i i i)$ to $(v)$. The remaining properties of Lemma 2.1 do not hold since sweeping is undone by resweeping, which is similar but not identical to sweeping. The sweep operator differs from Equation (2.2) by minus signs in the upper part and gives $-M^{-1}$ after sweeping on $N$ while partial inversion on $N$ gives $M^{-1}$. If $N$ is partitioned into more than two components, then the definition of both these operators still applies component by component.

Property $(v)$, written here explicitly for partial inversion of $M$ on $b$, is a standard equality for partitioned inverses:

$$
\left(\begin{array}{rr}
M_{a a . b} & M_{a-b} \\
-M_{b \vdash a} & M_{b b}^{-1}
\end{array}\right)=\left(\begin{array}{rr}
\left(M^{a a}\right)^{-1} & -\left(M^{a a}\right)^{-1} M^{a b} \\
-M^{b a}\left(M^{a a}\right)^{-1} & M^{b b . a}
\end{array}\right) .
$$

Compact explicit forms for the partitioned inverse result from Equation (2.3) and the basic properties $(i v)$ and $(v)$ of partial inversion, such as

$$
M^{a a}=M_{a a . b}^{-1}, \quad-M^{a b}=M_{a a . b}^{-1} M_{a \rightarrow b}=M_{a \leftarrow b} M_{b b . a}^{-1} .
$$

Equation (2.5) permits us to introduce some further notation for components of partially inverted matrices. For instance, for $N=\{G, J\}$ with $G=\{a, b\}$ and $J=\{c, d\}$, component $(G, d)$ in $M_{G \vdash d}=M_{G G}^{-1} M_{G d}$ is

$$
\left(\begin{array}{rr}
M_{a a . b}^{-1} & -M_{a a . b}^{-1} M_{a \neg b} \\
-M_{b b . a}^{-1} M_{b \rightarrow a} & M_{b b . a}^{-1}
\end{array}\right)\left(\begin{array}{c}
M_{a d} \\
M_{b d}
\end{array}\right)=\left(\begin{array}{c}
M_{a a . b}^{-1} M_{a d . b} \\
M_{b b . a}^{-1} M_{b d . a}
\end{array}\right)=\left(\begin{array}{c}
M_{a-d . b} \\
M_{b-d . a}
\end{array}\right)
$$


and component $(d, G)$ is

$$
M_{d \rightarrow G}=\left(\begin{array}{lll}
M_{d a . b} M_{a a . b}^{-1} & M_{d b . a} M_{b b . a}^{-1}
\end{array}\right)=\left(\begin{array}{ll}
M_{d . b \neg a} & M_{d . a \neg b}
\end{array}\right),
$$

where, e.g., $M_{a d . b}=M_{a d}-M_{a b} M_{b b}^{-1} M_{b d}$. The notation $M_{b-d . a}$ reminds us that partial inversion has first been carried out on $a$, then by additional partial inversion on $b$, the component $(b, d)$ is the matrix $M_{b d . a}$ multiplied to the left by $M_{b b . a}^{-1}$. Similarly, $M_{d . a \rightarrow b}$ is the matrix $M_{d b . a}$ multiplied to the right by $M_{b b . a}^{-1}$.

For $N=\{a, K\}$ with $K=\{b, c, d\}$, the change of partial inversion on $a$ to partial inversion on $\{a, b\}=G$ can now be studied in Theorem 2.2 and Corollary 2.3 below by using the following types of compact expressions for the resulting matrices

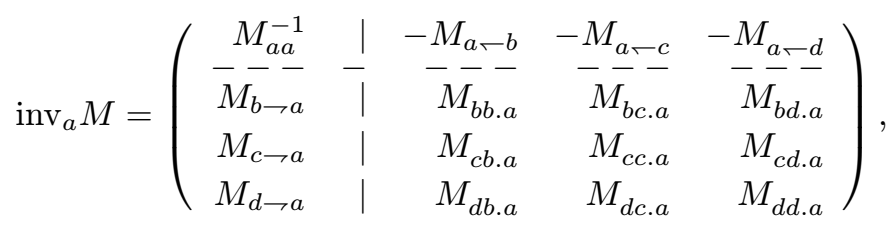

and, by using Equation (2.5), we get

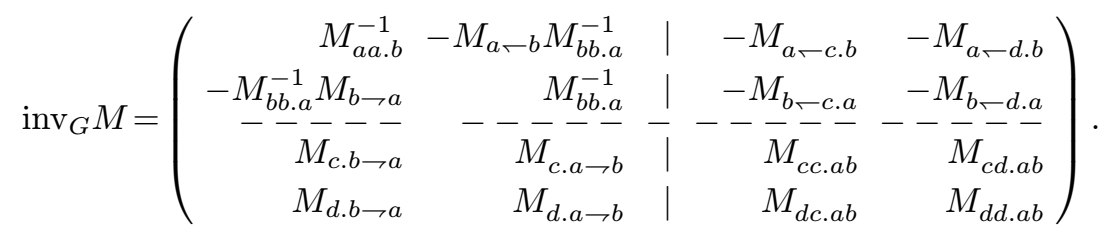

\subsection{Main derived properties.}

Now the main properties of partial inversion can be summarized.

Theorem 2.2. Commutativity, exchangeability and symmetric difference for partial inversion. Let arbitrary components $a, b, c$ partition $N, G=\{a, b\}$, and the matrix $M$ be accordingly partitioned, then

$$
\begin{aligned}
\text { (i) } \quad \operatorname{inv}_{a} \operatorname{inv}_{b} M & =\operatorname{inv}_{b} \operatorname{inv}_{a} M=\operatorname{inv}_{G} M \\
\text { (ii) } \quad\left[\operatorname{inv}_{a} M\right]_{G, G} & =\operatorname{inv}_{a} M_{G G} ; \\
\text { (iii) } \operatorname{inv}_{a b} \operatorname{inv} b c & =\operatorname{inv}_{a c} M .
\end{aligned}
$$

Proof. The commutativity in case $(i)$ results with the change from Equation (2.8) to Equation (2.9) and properties (iii) and ( $i v)$ in Lemma 2.1. The exchangeability in case $(i i)$ of a submatrix chosen after partial inversion and partial inversion carried out on a submatrix, is a consequence of property $(i)$ in this Theorem and of the definition in Equation (2.2) of the operator. The symmetric difference property (iii) results from the cases $(i)$, both in this Theorem and in Lemma 2.1. 
Illustration 2.1. For a square matrix $M$ of dimension $d_{N}=3$ and elements $m_{i j}$ partial inversion on $a=\{1,2\}$, carried out in two steps by starting with row and column 1, gives directly from Equation (2.2)

$$
\operatorname{inv}_{1} M=\left(\begin{array}{r|rr}
1 / m_{11} & -m_{12} / m_{11} & -m_{13} / m_{11} \\
---- & ----- & ---- \\
m_{21} / m_{11} & m_{22.1} & m_{23.1} \\
m_{31} / m_{11} & m_{32.1} & m_{33.1}
\end{array}\right)
$$

Partially inverting this matrix on row and column 2, using Equation (2.5) and exploiting case $(i)$ of Theorem 2.2, gives the compact expression

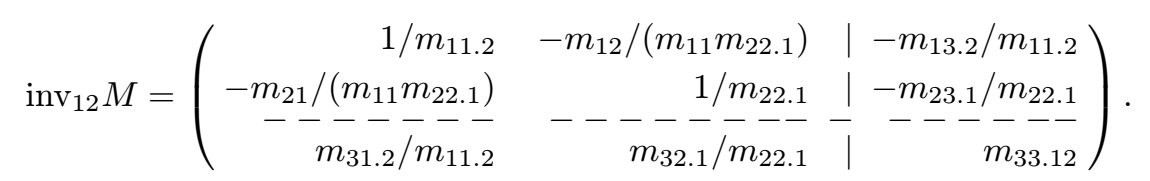

\subsection{Recursion relations.}

General recursion relations result from explicit expressions of the matrices obtained after successive steps of partial inversion.

Corollary 2.3. Recursion relations obtained by partial inversion. Let the subsets $a, b, c, d$ partition $N$ and the matrix $M$ be accordingly partitioned. Let further $G=\{a, b\}, H=\{a, b, c\}, J=\{c, d\}$ and $K=\{b, c, d\}$. Then, when $M$ is partially inverted in sequence on $a, b, c$, there results

(i) for elements corresponding to $(c, d)$ in $M_{K K . a}$ and in $M_{J J . G}$

$$
M_{c d . a}=M_{c d}-M_{c a} M_{a a}^{-1} M_{a d}, \quad M_{c d . a b}=M_{c d . a}-M_{c b . a} M_{b b . a}^{-1} M_{b d . a} ;
$$

(ii) for elements corresponding to $(a, d)$ in $M_{G-J}$ and in $M_{H \vdash d}$

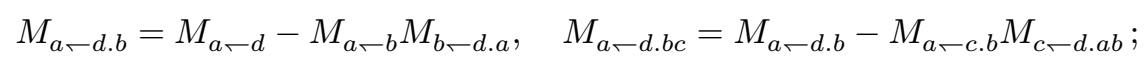

(iii) for elements corresponding to $(d, a)$ in $M_{J \rightarrow G}$ and in $M_{d \rightarrow H}$

$$
M_{d . b \rightarrow a}=M_{d \rightarrow a}-M_{d . a \rightarrow b} M_{b \rightarrow a}, \quad M_{d . b c \rightarrow a}=M_{d . b \rightarrow a}-M_{d . a b \rightarrow c} M_{c . b \rightarrow a} ;
$$

(iv) for elements corresponding to $(a, a)$ in $M_{G G}^{-1}$ and in $M_{H H}^{-1}$

$$
M_{a a . b}^{-1}=M_{a a}^{-1}-M_{a \leftarrow b} M_{b b . a}^{-1} M_{b \rightarrow a}, \quad M_{a a . b c}^{-1}=M_{a a . b}^{-1}-M_{a \leftarrow c . b} M_{c c . a b}^{-1} M_{c . b \neg a} .
$$

ProOF. The relations result by interpreting the modifications due to repeated partial inversion. The forms of $M_{c d . a b}, M_{a-d . b}, M_{d . b \rightarrow a}$, and $M_{a a . b}^{-1}$ are components when changing from $\operatorname{inv}_{a} M$ in Equation (2.8) to $\operatorname{inv}_{b} \operatorname{inv}_{a} M$ in Equation (2.9). Similarly, $M_{a \leftarrow d . b c}, M_{d . b c \rightarrow a}$, and $M_{a a . b c}^{-1}$ are components when changing from $\operatorname{inv}_{G} M$ in Equation (2.9) to $\operatorname{inv}_{c} \operatorname{inv}_{G} M$. 


\subsection{Directly related matrix decompositions.}

Direct computations show also that rows and columns of the matrices in a block-triangular decomposition $M=\mathrm{L} K \backslash$ can be specified in terms of partial inversion. Here, $K$ is a block-diagonal matrix, $\mathrm{L}$ is a unit lower block-triangular matrix and $\urcorner$ is a unit upper block-triangular matrix. For the following Lemma and throughout the paper let $d$ arbitrary subsets of $N$ be given. When these are ordered as $(1, \ldots, g, \ldots, d)$, then we call the result an ordered partitioning of $N$.

LEMMA 2.4. The relation of block-triangular decompositions to partial inversion. Let an ordered partitioning $(1, \ldots, g, \ldots, d)$ of $N$ be given, and $M=\mathrm{L} K \backslash$ be a corresponding block-triangular decomposition. Let $r$ denote all indices to the right and $l$ all indices to the left of $g$, both excluding $g$. Furthermore, let $\mathrm{L}_{N g}$ be columns of $\mathrm{L}$ and $\overline{\mathrm{T}}_{\mathrm{gN}}$ be the rows of $\mathrm{T}$, both corresponding to $\mathrm{g}$. Then

$$
\mathrm{L}_{N g}=\left(\begin{array}{r}
0_{l g} \\
I_{g g} \\
M_{r . l \rightarrow g}
\end{array}\right), \quad K_{g g}=M_{g g . l}, \quad \neg_{g N}=\left(0_{g l} I_{g g} M_{g-r . l}\right) .
$$

Thereby we use the convention that the submatrix of indices $(l, g)$ is absent when $g=1$ and the submatrix of $(g, r)$ is absent when $g=d$.

Illustration 2.2. For instance, for $d=3$ and the three blocks denoted by $a, b, c$, the decomposition is

$$
M=\left(\begin{array}{rrr}
I_{a a} & 0 & 0 \\
M_{b \rightarrow a} & I_{b b} & 0 \\
M_{c \rightarrow a} & M_{c . a \rightarrow b} & I_{c c}
\end{array}\right)\left(\begin{array}{rrr}
M_{a a} & 0 & 0 \\
0 & M_{b b . a} & 0 \\
0 & 0 & M_{c c . a b}
\end{array}\right)\left(\begin{array}{rrr}
I_{a a} & M_{a \leftarrow b} & M_{a \leftarrow c} \\
0 & I_{b b} & M_{b \vdash c . a} \\
0 & 0 & I_{c c}
\end{array}\right) .
$$

The form of $M$ with the partitioning refined so that each block contains a single element, leads to the following result which is closely related to those given recently under slightly weaker assumptions [21]. We denote e.g. by $\mathrm{L}^{-\mathrm{T}}$ the transpose of the inverse of matrix L.

LEMma 2.5. Decomposition of $M$ into a symmetric and a unit triangular matrix. A square matrix $M$, whose principal submatrices are all invertible, can be decomposed into the invertible symmetric matrix $S=\mathrm{L} K \mathrm{~L}^{\mathrm{T}}$ and the unit upper-triangular matrix $\left.\urcorner=\mathrm{L}^{-\mathrm{T}}\right\urcorner$ chosen so that $\left.M=S\right\urcorner$. In addition, there is the decomposition $\left.S^{*}=\right\urcorner^{\mathrm{T}} K \backslash$ and the unit lower-triangular matrix $L=\mathrm{L}^{-\mathrm{T}}$ chosen so that $M=L S^{*}$, where $\mathrm{L}$, 7 and $K$ are given by Equation (2.10) for $d=d_{N}$.

Proof. For $d=d_{N}$, the matrix $\mathrm{L}$ in the decomposition given in Equation (2.10) is unit lower triangular, $K$ is diagonal, and $\urcorner$ is unit upper-triangular. The inverse $\mathrm{L}^{-\mathrm{T}}$ of $\mathrm{L}^{\mathrm{T}}$ is unit upper-triangular and the product of two unit uppertriangular matrices is of the same form. There is a similar argument for 7 .

The decomposition of Equation (2.10) applied to a symmetric matrix $M$ has \urcorner$=\mathrm{L}^{\mathrm{T}}$, so that $M=\mathrm{L} K T$ in Lemma 2.5 reduces with $S=S^{*}$ to the usual 
triangular decomposition of a positive definite matrix. There is also the interpretation of $\mathrm{L}^{-1}$ as block-triangularizing $M$ from the left and of $7^{-1}$ as blocktriangularizing $M$ from the right with

$$
\left.\left.{ }^{\prime} M=\mathrm{L}^{-1} M=K\right\urcorner, \quad M^{\prime}=M\right\urcorner^{-1}=\mathrm{L} K,
$$

so that ' $M$ is upper block-triangular and $M^{\prime}$ is lower block-triangular.

Now, the elements of the block-triangular and triangular decompositions of an invertible symmetric matrix permit an interpretation in terms of partial inversion, which is to be given in Theorem 3.1 in the following section.

\section{Some direct applications to linear statistical models.}

\subsection{Elements of partially inverted covariance matrices.}

Let $\Sigma$ be the invertible covariance matrix of a mean-centered column vector random variable $Y$, and let $\Sigma^{-1}$ be the concentration matrix of $Y$. Let further a split of $Y$ be defined by two arbitrary vector components, $Y_{a}$ and $Y_{b}$. Then, Lemma $2.1(v)$ equates two nonsymmetric matrices

$$
\operatorname{inv}_{a} \Sigma^{-1}=\left(\begin{array}{rr}
\left(\Sigma^{a a}\right)^{-1} & -\left(\Sigma^{a a}\right)^{-1} \Sigma^{a b} \\
\sim & \Sigma^{b b . a}
\end{array}\right)=\left(\begin{array}{rc}
\Sigma_{a a \mid b} & \Sigma_{a b} \Sigma_{b b}^{-1} \\
\sim & \Sigma_{b b}^{-1}
\end{array}\right)=\operatorname{inv}_{b} \Sigma
$$

Here, and throughout, the $\sim$ notation indicates entries in a matrix which is symmetric up to the sign, i.e. minus elements given in the upper off-diagonal part of the matrix.

The off-diagonal matrices specify two different, but equivalent, ways of computing $\Pi_{a \mid b}$, the matrix of regression coefficients of $Y_{b}$ in linear least squares regression of $Y_{a}$ on $Y_{b}$ [11]. This coefficient matrix is defined by the linear equation $Y_{a}=\Pi_{a \mid b} Y_{b}+\varepsilon_{a}$ in which $\operatorname{cov}\left(\varepsilon_{a}, Y_{b}^{\mathrm{T}}\right)=0$, i.e. with

$$
\Sigma_{a b}=E\left(Y_{a} Y_{b}^{\mathrm{T}}\right)=\Pi_{a \mid b} E\left(Y_{b} Y_{b}^{\mathrm{T}}\right)+E\left(\varepsilon_{a} Y_{b}^{\mathrm{T}}\right)=\Pi_{a \mid b} \Sigma_{b b}
$$

The interpretation of $\Sigma^{b b . a}=\Sigma_{b b}^{-1}$ in Equation (3.1) as the concentration matrix of $Y_{b}$ and of $\left(\Sigma^{a a}\right)^{-1}=\Sigma_{a a \mid b}$ as the covariance matrix of $Y_{a \mid b}=Y_{a}-\Pi_{a \mid b} Y_{b}$ had been derived by Dempster [13] in terms of the sweep operator for symmetric matrices. In this context, we denote Schur matrices that result from marginalizing by $\Sigma^{b b . a}$, and those obtained from conditioning by $\Sigma_{a a \mid b}$.

With subsets $a, b, c, d$ partitioning $N, G=\{a, b\}$ and $J=\{c, d\}$, we denote the different components of least squares regression coefficient matrices by

$$
\Pi_{G \mid J}=\left(\Pi_{G \mid c . d} \Pi_{G \mid d . c}\right), \quad \Pi_{G \mid J}=\left(\begin{array}{c}
\Pi_{a \mid J} \\
\Pi_{b \mid J}
\end{array}\right),
$$

so that, for instance, $\Pi_{b \mid c . d}$ contains the coefficients of $Y_{c}$ in a least squares regression of $Y_{b}$ on both $Y_{c}$ and $Y_{d}$. Matrix forms of recursion relations for least squares regression coefficients [7], for covariances [1], and for concentrations [13], 
are then recognized to be consequences of the recursion properties of partial inversion and are

$$
\begin{aligned}
\Pi_{b \mid d . c} & =\Pi_{b \mid d}-\Pi_{b \mid c . d} \Pi_{c \mid d} \\
\Sigma_{b d \mid c} & =\Sigma_{b d}-\Sigma_{b c} \Sigma_{c c}^{-1} \Sigma_{c d} \\
\Sigma^{b d . a} & =\Sigma^{b d}-\Sigma^{b a}\left(\Sigma^{a a}\right)^{-1} \Sigma^{a d} .
\end{aligned}
$$

Equations (3.3) provide insight into when regression coefficients, covariances and concentrations remain unchanged after marginalizing or after conditioning. Elements in positions $(i, j)$ of $\operatorname{inv}_{G} \Sigma^{-1}$ are proportional to different types of partial correlation, compare e.g. [1], Sections 2.3, 2.5, [10], Section 3.4, denoted here by

(i) $\rho_{i j \mid J \backslash j} \quad$ for $\Pi_{G \mid J}$ with $i \in G, j \in J$;

(ii) $\rho_{i j \mid J} \quad$ for $\Sigma_{G G \mid J}$ with $i \in G, j \in G$;

(iii) $\rho_{i j \mid J \backslash\{i j\}}$ for $\Sigma^{J J . G}$ with $i \in J, j \in J$.

Equations (3.4) give the interpretation of different types of edge to represent an independence structure in $\operatorname{inv}_{G} \Sigma^{-1}$, arrows for $\Pi_{G \mid J}$ and two different types of undirected graph for $\Sigma_{G G \mid J}$, called a conditional covariance graph, and in $\Sigma^{J J . G}$, called a marginal concentration graph, see [10,44].

Equations (3.4) and (3.3) jointly capture also how probabilistic independence statements combine for Gaussian distributions that are nondegenerate, i.e. for which $\Sigma$ is positive definite. With $Y_{b}$ independent of $Y_{d}$ given $Y_{c}$ denoted by $b \Perp d \mid c[12]$,

(i) $\quad b \Perp d \quad$ and $\quad(b \Perp c \mid d$ or $c \Perp d)$ imply $b \Perp d \mid c$;

(ii) $b \Perp d \quad$ and $\quad(b \Perp c$ or $c \Perp d)$ imply $b \Perp d \mid c$;

(iii) $b \Perp d \mid a c \quad$ and $\quad(b \Perp a \mid c d$ or $d \Perp a \mid b c)$ imply $b \Perp d \mid c$.

\subsection{Relations to triangular decompositions of $\Sigma$ and of $\Sigma^{-1}$.}

Partial inversion applied repeatedly to symmetric matrices leads in particular to the following interpretations of the resulting matrix components.

THEOREM 3.1. Interpretation of block-triangular decompositions of invertible $\Sigma$ and $\Sigma^{-1}$. Let an ordered partitioning $(1, \ldots, g, \ldots, d)$ of $N$ be given. Let further $r$ denote all indices to the right and $l$ all indices to the left of $g$, excluding $g$ in this new ordering. The unit block-triangular decompositions is $\left.\left.\Sigma^{-1}=\right\urcorner^{\mathrm{T}} H\right\urcorner$, compare Illustration 2.2 , and $\Sigma=L^{\mathrm{T}} K L$. Then

$$
\begin{aligned}
\text { (i) } H & =K^{-1} \quad \text { and } \quad L^{\mathrm{T}}=7^{-1} ; \\
(\text { ii }) \quad H_{g g} & =\Sigma^{g g . l}, \quad \neg_{g N}=\left(\begin{array}{lll}
0_{g l} I_{g g}-\Pi_{g \mid r}
\end{array}\right) ; \\
(\text { iii }) & K_{g g}=\Sigma_{g g \mid r}, \quad L_{g N}=\left(\Pi_{l \mid g . r}^{\mathrm{T}} I_{g g} 0_{g r}\right) ;
\end{aligned}
$$


where $\Sigma^{g g . l}$ and $\Sigma_{g g \mid r}$ are the concentration matrix and the covariance matrix of $Y_{g \mid r}$.

Illustration 3.1. For instance, for $d=4$, the lower block-triangular decomposition $(H\urcorner$,$) of \Sigma^{-1}$ is

$$
H=\left(\begin{array}{rrrr}
\Sigma^{a a} & 0 & 0 & 0 \\
. \Sigma^{b b . a} & 0 & 0 \\
. & . & \Sigma^{c c . a b} & 0 \\
. & . & . & \Sigma^{d d . a b c}
\end{array}\right), \quad T=\left(\begin{array}{rrrr}
I_{a a} & -\Pi_{a \mid b . c d} & -\Pi_{a \mid c . b d} & -\Pi_{a \mid d . b c} \\
0 & I_{b b} & -\Pi_{b \mid c . d} & -\Pi_{b \mid d . c} \\
0 & 0 & I_{c c} & -\Pi_{c \mid d} \\
0 & 0 & 0 & I_{d d}
\end{array}\right) \text {, }
$$

where, here, and throughout, the . notation indicates entries in a symmetric matrix, i.e. elements given in the upper off-diagonal part of the matrix. For the block-triangular decomposition $\left(K, \mathrm{~L}^{\mathrm{T}}\right)$ of $\Sigma$ we have

$$
K=\left(\begin{array}{rrrr}
\Sigma_{a a \mid b c d} & 0 & 0 & 0 \\
. \Sigma_{b b \mid c d} & 0 & 0 \\
. & . & \Sigma_{c c \mid d} & 0 \\
. & . & . & \Sigma_{d d}
\end{array}\right), \quad \mathrm{L}=\left(\begin{array}{rrrr}
I_{a a} & 0 & 0 & 0 \\
\Pi_{a \mid b . c d}^{\mathrm{T}} & I_{b b} & 0 & 0 \\
\Pi_{a \mid c . d}^{\mathrm{T}} & \Pi_{b \mid c . d}^{\mathrm{T}} & I_{c c} & 0 \\
\Pi_{a \mid d}^{\mathrm{T}} & \Pi_{b \mid d}^{\mathrm{T}} & \Pi_{c \mid d}^{\mathrm{T}} & I_{d d}
\end{array}\right) .
$$

Proof. Case $(i)$ of Theorem (3.1) is direct by matrix inversion. Case $(i i)$ is proven by Lemma 2.4. Furthermore, since the concentration matrix of $Y_{b}$ has with $\Sigma^{b b . a}$ the same form as $\Sigma^{-1}$, the same type of argument applies to it and, similarly, to $\Sigma^{g g . l}$. In this way, the block-triangular decomposition of Equation (2.10) is built up for $\Sigma^{-1}$. To prove case $(i i i)$, we note that the form of the block-triangular decomposition of $\Sigma$ results by matrix inversion from case (ii) and by the recursion relations for regression coefficients in Equation (3.3).

\subsection{Relations to triangularized forms of $\Sigma$ and of $\Sigma^{-1}$.}

With a given ordered partitioning $N=(1, \ldots, g, \ldots, d)$ block $g$ is associated with the vector variable $Y_{g}$. For such a sequence of vector variables we denote the left-triangularized form of the concentration matrix by ${ }^{\prime} \Sigma^{-1}$ and the right-triangularized form of the covariance matrix by $\Sigma^{\prime}$, and derive next their components after partial inversion.

COROLlary 3.2. Interpretation of partial inversion for ${ }^{\prime} \Sigma^{-1}$ and $\Sigma^{\prime}$. Let the matrices ${ }^{\prime} \Sigma^{-1}=H \backslash$ and $\Sigma^{\prime}=K \mathrm{~L}$ be defined, from the block-triangular decompositions of Theorem 3.1, for an invertible covariance matrix $\Sigma$. Then,

$$
\begin{aligned}
(i) & {\left[{ }^{\prime} \Sigma^{-1}\right]_{g, N} } & =\left(\begin{array}{lll}
0_{g l} & \Sigma^{g g . l} & \Sigma^{g r . l}
\end{array}\right) ; \\
(\text { ii }) & \Sigma_{g N}^{\prime} & =\left(\begin{array}{lll}
\Sigma_{g l \mid r} & \Sigma_{g g \mid r} & 0_{g r}
\end{array}\right) ; \\
(\text { iii }) & {\left[\operatorname{linv}_{l g}{ }^{\prime} \Sigma^{-1}\right]_{g, N} } & =\left(\begin{array}{lll}
0_{g l} & \Sigma_{g g \mid r} & \Pi_{g \mid r}
\end{array}\right),
\end{aligned}
$$

where $\Sigma^{\text {gr.l }}$ gives the concentrations of $Y_{g}$ and $Y_{r}$, while $\Sigma_{g l \mid r}$ gives the covariances of $Y_{g \mid r}$ and $Y_{l \mid r}$; all other submatrices are as defined for Theorem 3.1. 
Illustration 3.2. For $d=4$, the left block-triangularized matrix ${ }^{\prime} \Sigma^{-1}$ in (i) and the right-triangularized matrix $\Sigma^{\prime}$ in $(i i)$ are

$$
{ }^{\prime} \Sigma^{-1}=\left(\begin{array}{rrrr}
\Sigma^{a a} & \Sigma^{a b} & \Sigma^{a c} & \Sigma^{a d} \\
0 & \Sigma^{b b . a} & \Sigma^{b c . a} & \Sigma^{b d . a} \\
0 & 0 & \Sigma^{c c . a b} & \Sigma^{c d . a b} \\
0 & 0 & 0 & \Sigma^{d d . a b c}
\end{array}\right), \Sigma^{\prime}=\left(\begin{array}{rrrr}
\Sigma_{a a \mid b c d} & 0 & 0 & 0 \\
\Sigma_{b a \mid c d} & \Sigma_{b b \mid c d} & 0 & 0 \\
\Sigma_{c a \mid d} & \Sigma_{c b \mid d} & \Sigma_{c c \mid d} & 0 \\
\Sigma_{d a} & \Sigma_{d b} & \Sigma_{d c} & \Sigma_{d d}
\end{array}\right) .
$$

The matrices of case (iii) are, for this example of four blocks, the matrices $K$ and 7 in equations (3.6) and (3.7).

Proof. From the block-triangular decomposition $\left.\left.\Sigma^{-1}=\right\urcorner^{\mathrm{T}} H\right\urcorner$, where $\urcorner$ is unit upper block-triangular, the matrix ${ }^{\prime} \Sigma^{-1}=H \backslash$ is upper block-triangular. The interpretation in $(i)$ follows from the product $H \backslash$ by using the definition of least-squares regression coefficient matrices in terms of concentrations. A similar argument applies to the block-triangular decomposition $\Sigma=\mathrm{L}^{\mathrm{T}} K \mathrm{~L}$, where $\mathrm{L}=7^{-\mathrm{T}}$ is unit lower block-triangular, by using the definition of least-squares regression coefficient matrices in terms of covariances for the product $K \mathrm{~L}$.

The upper block-triangularity of ' $\Sigma^{-1}$, the definition of partial inversion in Equation (2.2), and the exchangeability property in Theorem 2.2 case $(i i)$, imply for $\bar{l}=N \backslash l$ that

$$
\left[\operatorname{inv}_{1, \ldots, g^{\prime}} \Sigma^{-1}\right]_{\bar{l}, \bar{l}}=\operatorname{inv}_{g}\left[{ }^{\prime} \Sigma^{-1}\right]_{\bar{l}, \bar{l}}=\left(\begin{array}{cc}
\Sigma_{g g \mid r} & \Pi_{g \mid r} \\
0_{r g} & { }^{\prime} \Sigma^{r r . l g}
\end{array}\right)
$$

and hence the form given in $(i i i)$.

\section{Relations to linear graphical chain models.}

The partial inversion results in the previous section relate directly to linear stepwise data generating processes and to different types of statistical joint response models with independence constraints, all generated by the directed acyclic graph associated with the generating process.

\subsection{Relations to linear triangular systems.}

With a partitioning of $N$, refined to contain only single elements, one obtains from Theorem 3.1 (ii) a triangular decomposition of $\Sigma^{-1}$, where 7 is uppertriangular and $H=\Delta^{-1}$ is a diagonal matrix with all diagonal elements positive. This gives the parameters in a stepwise generating process for a covariance matrix which has been called a path analysis model by the geneticist Wright $[46,47]$, a system of linear recursive equations with uncorrelated residuals by the econometrician Wold [45], or, more recently, a linear triangular system [44].

For a mean-centered random column vector $Y$ and ordering $\left(1, \ldots, d_{N}\right)$, such a process can be written in matrix notation as

$$
A Y=\varepsilon, \quad \text { with } \operatorname{cov}(\varepsilon)=\Delta,
$$


having

$$
\Sigma=\operatorname{cov}(Y)=A^{-1} \Delta A^{-\mathrm{T}}, \quad \text { and } \quad \Sigma^{-1}=\operatorname{con}(Y)=A^{\mathrm{T}} \Delta^{-1} A .
$$

In our notation, $A$ is unit upper-triangular, so that from Theorem 3.1, see also 7 and $\mathrm{L}^{\mathrm{T}}$ of Illustration 3.1, the elements of $a_{i j}$ of $A$ are

$$
a_{i j}=-\beta_{i \mid j . r(i) \backslash j},
$$

where $\beta_{i \mid j . C}$ denotes the coefficient of $Y_{j}$ in linear least squares regression of $Y_{i}$ on $Y_{j}$ and $Y_{C}$. Here, $C$ may refer to a vector variable $Y_{C}$ and $r(k)=\{k+1$, $\ldots, d\}$. The diagonal elements of $\Delta$ are $\delta_{i i}=\sigma_{i i \mid r(i)}$, the residual variances in the corresponding linear regressions.

When $(1, \ldots, d)$ refers to a time order, then $Y_{1}$ is the most recent response variable and $Y_{d}$ is the variable in the past, being most distant from it. The joint concentration matrix is directly generated by a sequence of univariate least squares regressions with $Y_{i}$ as response to $Y_{i+1}, \ldots, Y_{d}$. Expressed differently, $A$ contains the generating equation parameters and $\left(A, \Delta^{-1}\right)$ gives the unique triangular decomposition of $\Sigma^{-1}$ for one fixed order of the variables.

When there is zero contribution of a potentially explanatory variable $Y_{j}$ for $Y_{i}$, this is represented by a zero value in position $(i, j)$ in the upper triangular part of $A$. Variables with a nonzero contribution are called the parents of $i$, denoted by $\operatorname{par}(i)$. Equation (4.2) represents then an unconstrained model containing as a special case the reduced model [8] with

$$
a_{i j}=-\beta_{i \mid j \cdot \operatorname{par}(i) \backslash j} \quad \text { when } j \in \operatorname{par}(i), \quad 0=\rho_{i j \mid \operatorname{par}(i)}=\rho_{i j \mid r(i) \backslash j} \quad \text { else. }
$$

For the more general model with parameters (4.2) and for the constrained parameters (4.3), explicit least-squares estimates are available. In each application this permits direct checks of the goodness of fit of the hypothesized constraints.

By $\operatorname{In}[M]$ we denote the indicator matrix of a matrix $M$. It is obtained by replacing every nonzero element of $M$ by a one. The edge matrix of the generating graph is $\mathcal{A}=\operatorname{In}[A]$, where node $i$ corresponds to variable $Y_{i}$. The graph is often called the parent graph since it shows the directly explanatory variables of $Y_{i}$ by arrows starting in the parent node set $\operatorname{par}(i)$ and pointing to node $i$. It defines also the linear independence structure in a family of matrices in which the unconstrained equation parameters $a_{i j}$ in Equation (4.3) are free to vary, provided only that they lead to positive residual variances.

\subsection{Relations to joint response models.}

Sequences of joint responses occur in different types of graphical chain models, used to study multivariate statistical dependence. These have been defined for more general than linear relations, but we discuss here mainly the linear case and introduce some more terminology first. All graphical chain models have in common that the variables are arranged in a sequence of, say $d$, chain components. Each component $g$ contains one or more variables. Several variables in the same chain component are considered as joint responses, so that within chain 
components there are undirected associations of interest and between chain components there are directed associations. Each missing edge in an associated chain graph captures a conditional independence constraint and each edge present corresponds to a conditional association. The precise conditioning set depends on the type of chain graph.

For $d=1$, models with zero constraints on $\Sigma^{-1}$ have been introduced as covariance selection models by Dempster [14]. Corresponding recursive sequences of such models for $1<d<d_{N}$ are blocked-concentration chains, defining ${ }^{\prime} \Sigma^{-1}$ $[28,19,41,38]$.

For $d=1$, models with zero constraints on $\Sigma$ have been introduced as hypotheses linear in covariances by Anderson $[2,3]$ and studied later as independence models $[26,35]$; we call corresponding recursive sequences of such models for $1<d<d_{N}[25]$ blocked-covariance chains defining ' $\Sigma$.

Models with zero constraints on $H=\Sigma_{g g \mid r(g)}$ and $\Pi_{g \mid r}$ are multivariate regression chains, which include seemingly unrelated regressions $[9,10,35]$. We speak of concentration-regression chains for models with zero constraints on elements of either component of the block-triangular decomposition $(H\urcorner$,$) of \Sigma^{-1}$. These have been studied by Perlman and coauthors, [4], while the partial regression chains with zero constraints on the block-triangular decomposition $\left(K, \mathrm{~L}^{\mathrm{T}}\right)$ of $\Sigma$ appear to have not yet been studied by statisticians.

Matrix relations between parameters in linear chain graph model to those of a generating linear triangular system [44] are not repeated here, instead, the relations to partial inversion are spelled out.

COROLlary 4.1. Induced joint response models related to partial inversion. Let $(1, \ldots, g, \ldots, d)$ be an ordered partitioning of $N$, defining block decompositions $\Sigma=\mathrm{L}^{\mathrm{T}} K \mathrm{~L}$ and $\Sigma^{-1}=\mathrm{\top}^{\mathrm{T}} H \mathrm{~T}$ of Theorem 3.1, and $\left.{ }^{\prime} \Sigma^{-1}=H\right\urcorner, \Sigma^{\prime}=K \mathrm{~L}$, then unconstrained parameters induced in

(i) ' $\Sigma^{-1}$, for a blocked concentration chain;

(ii) $H$ and $\urcorner$ for a concentration-regression chain;

(iii) $\Sigma^{\prime}$, for a blocked covariance chain;

(iv) $K$ and $L^{\mathrm{T}}$ for a partial regression chain;

(iv) $K$ and $\urcorner$, for a multivariate regression chain,

are in one-to-one correspondence to unconstrained specific parameters $A, \Delta$ of a given saturated triangular system and are obtainable by partial inversion.

Proof. It is implied by the partial inversion results of Theorem 5.1 and Corollary 3.2 that each of the sets of parameters is obtained by a one-to-one transformation of the covariance matrix.

One important consequence of Corollary 4.1 for statistical analysis is, that except possibly in a subset of lower dimension - the unconstrained parameters, estimated by equating observed to expected moments, are in one-to-one correspondence for the different types of saturated models. Also, from Equation (3.4), we know the corresponding conditional independence statement when a parameter is constrained to be zero for a joint Gaussian distribution. And, more impor- 
tantly, this provides the interpretation of each missing edge in all of the different types of chain graphs in general [44].

\section{Induced edge matrices corresponding to partial inversion.}

For the study of when and how independence constraints of a given stepwise generating process are preserved after partial inversion, we introduce an operator to find structural zeros for square, unit binary matrices $\mathcal{M}$ of dimension $d_{N}$. We denote by $\mathcal{M}_{a a}^{-}$a binary matrix which can be viewed as a generalized inverse of the binary matrix $\mathcal{M}_{a a}$. For this, the matrix

$$
\mathcal{M}_{a a}^{*}=\left(d_{N}+1\right) \mathcal{I}_{a a}-\mathcal{M}_{a a}
$$

may be chosen, where $\mathcal{I}$ denotes the identity matrix. It has the same zeros as $\mathcal{M}_{a a}$ and only nonnegative elements in its inverse

$$
\operatorname{In}\left[\mathcal{M}_{a a}^{*}\right]=\mathcal{M}_{a a} \text { and }\left(\mathcal{M}_{a a}^{*}\right)^{-1} \geq 0 .
$$

The reason is that $(\mathcal{I}-c \mathcal{M})^{-1}$ is the unique limit of the infinite geometric series $\mathcal{I}+c \mathcal{M}+c^{2} \mathcal{M}^{2}+\ldots$ when $c=1 /\left(d_{N}+1\right)$, see essentially [32, p. 29].

Analogously to (2.2), we write for structural zeros in $\operatorname{inv}_{a} M$, where the matrix $M$ has structural zeros in $\mathcal{M}$,

$$
\operatorname{zer}_{a} \mathcal{M}=\left(\begin{array}{cr}
\mathcal{M}_{a a}^{-} & \mathcal{M}_{a-b} \\
\mathcal{M}_{b \rightarrow a} & \mathcal{M}_{b b . a}
\end{array}\right)=\operatorname{In}\left(\begin{array}{cr}
\left(\mathcal{M}_{a a}^{*}\right)^{-1} & \mathcal{M}_{a a}^{-} \mathcal{M}_{a b} \\
\mathcal{M}_{b a} \mathcal{M}_{a a}^{-} & \mathcal{M}_{b b}+\mathcal{M}_{b a} \mathcal{M}_{a a}^{-} \mathcal{M}_{a b}
\end{array}\right)
$$

When $\mathcal{M}=\mathcal{A}$ is upper-triangular, it can be interpreted as the edge matrix of a parent graph which is directed and acyclic and has a full ordering of the nodes. When $\mathcal{M}=\mathcal{S}$ is symmetric, it can be the edge matrix of an undirected graph. The binary matrices $\mathcal{A}^{-}$and $\mathcal{S}^{-}$are then edge matrices of what is sometimes called the transitive closure of graphs with edge matrices $\mathcal{A}$ and $\mathcal{S}$, respectively. By applying Equation (5.1) repeatedly to each of the distinct rows and columns of $\mathcal{A}$ or $\mathcal{S}$, the edge matrix of the transitive closure of the graph is obtained. Therefore, the calculus applied to rows and columns $a$ of such an edge matrix can be interpreted as a method of finding the partial closure of the graph with respect to the nodes in set $a$.

The structural zero operator in Equation (5.1) cannot be undone when it is reapplied to the same rows and columns. The reason is that it is defined in terms of sums and products of nonnegative matrices so that zeros present in $\mathcal{M}$ may be preserved or removed but no new zeros can be generated. Therefore, the operator has the following properties.

Theorem 5.1. Commutativity, exchangeability and expansion for structural zeros preserved after partial inversion. Let arbitrary components $a, b, c, d$ partition $N, G=\{a, b\}$, and the matrix $\mathcal{M}$ be accordingly partitioned, then

$$
\begin{aligned}
\text { (i) } \quad \operatorname{zer}_{a} \operatorname{zer}_{b} \mathcal{M} & =\operatorname{zer}_{b} \operatorname{zer}_{a} \mathcal{M}=\operatorname{zer}_{G} \mathcal{M} ; \\
\text { (ii) } \quad\left[\operatorname{zer}_{a} \mathcal{M}\right]_{G, G} & =\operatorname{zer}_{a} \mathcal{M} G G ; \\
\text { (iii) } \operatorname{zer}_{a b} \operatorname{zer}_{b c} \mathcal{M} & =\operatorname{zer}_{a b c} \mathcal{M} .
\end{aligned}
$$


Whenever a chain graph model is generated by a linear triangular system, as stated in Corollary 4.1, then the variable pairs for which independence constraints are preserved in the induced joint response model may be obtained by using the structural zero operator.

To see this, suppose first that $\alpha, \beta, C$ are disjoint subsets of $N$, where only $C$ may be empty, and the dependence of $Y_{\alpha}$ on $Y_{\beta}$ given $Y_{C}$ is of interest. Then some variables $Y_{M}$ are implicitly omitted, and $N=(M, \alpha, \beta, C)$ becomes the relevant ordering of the node set. We let further $a=M \cup \alpha$ and $b=C \cup \beta$. Then, the relevant edge matrix components of the concentration matrix of $Y_{\alpha \mid C}$, $Y_{\beta \mid C}$, partially inverted with respect to $a$ [44], can be written in terms of the components of $\mathcal{B}=\operatorname{zer}_{a} \mathcal{A}$ as

$$
\begin{aligned}
\mathcal{S}_{\alpha \alpha \mid \beta C} & =\operatorname{In}\left[\mathcal{B}_{a a}\left(\mathcal{I}_{a a}+\mathcal{B}_{b a}^{\mathrm{T}} \mathcal{B}_{b a}\right)^{-} \mathcal{B}_{a a}^{\mathrm{T}}\right]_{\alpha, \alpha} ; \\
\mathcal{S}^{\beta \beta . \alpha M} & =\operatorname{In}\left[\mathcal{B}_{b b}^{T}\left(\mathcal{I}_{b b}+\mathcal{B}_{b a} \mathcal{B}_{a b}^{\mathrm{T}}\right)^{-} \mathcal{B}_{b b}\right]_{\beta, \beta} ; \\
\mathcal{P}_{\alpha \mid \beta . C} & =\operatorname{In}\left[\mathcal{B}_{a b}+\mathcal{B}_{a a} \mathcal{B}_{b a}^{\mathrm{T}}\left(\mathcal{I}_{b b}+\mathcal{B}_{b a} \mathcal{B}_{b a}^{\mathrm{T}}\right)^{-} \mathcal{B}_{b b}\right]_{\alpha, \beta} .
\end{aligned}
$$

Next, Equations (5.2) are used to find the independence structure in induced chain graphs with the $d$ components in $N=(1, \ldots, g, \ldots, d)$. For this, any pair of adjacent components is treated separately, by equating for instance $g$ with $\alpha$ and $g+1$ with $\beta$. Algorithms are available in the ggm package of $\mathrm{R}$ [30].

If $\mathcal{P}_{\alpha \mid \beta . C}=0$, then the generating process implies $\Pi_{a \mid b . C}=0$. For this judgement, it is not enough to just check in an application for a given set of parameters $A, \Delta$ whether $\Pi_{a \mid b . C}=0$, since this matrix may contain more zeros than there are structural zeros. Such additional zeros are due to parametric cancellation, i.e. to special relations between individual specific parameter values. More generally, the condition $\mathcal{P}_{\alpha \mid \beta . C}=0$ represents a matrix criterion for what has been called separation in directed acyclic graphs [33].

\section{Discussion.}

The statistical importance of the induced chain graphs, obtained with Equations (5.2), is that they apply to distributions of arbitrary form, provided that these distributions satisfy all independencies specified by the given parent graph and that these independencies may be combined as in a non-degenerate Gaussian distribution; see also [37].

Independence equivalence of two chain graphs means that the missing edges in the two graphs lead to the same set of independencies, i.e. specify an identical independence structure. For different special subclasses of chain graphs, criteria and algorithms to decide on independence equivalence have been derived. For instance, a concentration graph model can be independence equivalent to a triangular system if and only if the concentration graph is chordal, i.e. it contains no chordless cycle in 4 or more nodes. General results are still lacking for independence equivalence of the different types of chain graphs implied by Corollary 4.1. Criteria for chordality of graphs were given early, see e.g. [15], but 
efficient algorithms applicable to large graphs were derived only much later, see e.g. [29, 34].

Similarly, the explicit forms of edge matrices, generated by a given parent graph, need to be complemented by computationally efficient algorithms for applications to very large matrices and graphs. One general computational problem is to start with the zero structure in any given triangular decomposition of $\Sigma^{-1}$ and to obtain the implied structural zeros in a new block-triangular decomposition of $\Sigma^{-1}$ or of $\Sigma$, i.e. to find the zeros that are retained by corresponding symbolic matrix inversion. The problem is considerably more complex than checking for chordality of a concentration graph because of the block structure and because different components of block-triangular decompositions may require the combination of different types of edge.

\section{Acknowledgement.}

We thank the Swedish Research Society for support of the cooperation of D. R. Cox and N. Wermuth, as well as the German Research Society and the University of Mainz for research grants. We also thank Søren Johansen, Ingram Olkin and the referees for their thoughtful and constructive comments.

\section{REFERENCES}

1. T. W. Anderson, An Introduction to Multivariate Statistical Analysis, Wiley, New York, NY, 1958.

2. T. W. Anderson, Statistical inference for covariance matrices with linear structure, in Multivariate Analysis II, P. R. Krishnaiah (ed.), pp. 55-66, Academic Press, New York, 1969.

3. T. W. Anderson, Asymptotically efficient estimation of covariance matrices with linear structure, Ann. Stat., 1 (1973), pp. 135-141.

4. S. A. Andersson, D. Madigan, and M. D. Perlman, Alternative Markov properties for chain graphs, Scand. J. Stat., 28 (2001), pp. 33-86.

5. A. E. Beaton, The Use of Special Matrix Operators in Statistical Calculus, EdD. thesis, Harvard University, reprinted as ETS Research Bulletin 64-51, Educational Testing Service, Princeton, NJ, 1964.

6. A. L. Cholesky, Note sur une méthode de résolution des équations normales provenant de l'application de la méthode des moindres carrés à un sytème d'équations linéaires en nombre inférieur à celui des inconnues (Published six years after Cholesky's death by Benoit), Bull. Géodésique, 2 (1924), pp. 67-77.

7. W. G. Cochran, The omission or addition of an independent variate in multiple linear regression, Suppl. J. R. Stat. Soc., 5 (1938), pp. 171-176.

8. D. R. Cox and N. Wermuth, An approximation to maximum-likelihood estimates in reduced models, Biometrika, 77 (1990), pp. 747-761.

9. D. R. Cox and N. Wermuth, Linear dependencies represented by chain graphs (with discussion), Stat. Sci., 8 (1993), pp. 204-218; 247-277.

10. D. R. Cox and N. Wermuth, Multivariate Dependencies: Models, Analysis, and Interpretation, Chapman and Hall, London, 1996.

11. H. Cramér, Mathematical Methods of Statistics, Princeton University Press, Princeton, NJ, 1946. 
12. A. P. Dawid, Conditional independence in statistical theory, J. R. Stat. Soc., Ser. B, 41 (1979), pp. 1-31.

13. A. P. Dempster, Elements of Continuous Multivariate Analysis, Addison-Wesley, Reading, MA, 1969.

14. A. P. Dempster, Covariance selection, Biometrics, 28 (1972), pp. 157-175.

15. G. A. Dirac, On rigid circuit graphs, Abh. Math. Semin. Univ. Hamb., 25 (1961), pp. 7176.

16. M. H. Doolittle, Explanations and illustrations of method employed in the office in solution of normal equations and the adjustment of a triangulation, U.S. Coast and Geodetic Survey, Appendix 8 (1878), pp. 115-120.

17. D. Edwards, Introduction to Graphical Modelling, 2nd edn., Springer, New York, NY, 2000.

18. L. Fox, H. D. Huskey, and J. H. Wilkinson, Notes on the solutions of algebraic linear simultaneous equations, Quart. J. Mech. Appl. Math., 1 (1947), pp. 149-173.

19. M. Frydenberg, The chain graph Markov property, Scand. J. Stat., 17 (1990), pp. 333-353.

20. W. Gibbs, Elementary Principles of Statistical Mechanics, Yale University Press, New Haven, CT, 1902.

21. G. H. Golub and J. Y. Yuan, Symmetric-triangular decomposition and its applications Part I: Theorems and algorithms, BIT, 42 (2002), pp. 814-822.

22. J. P. Gram, Über die Entwickling reeller Funktionen in Reihen mittelst der Methode der kleinsten Quadrate, Crelle's Journal (J. Reine Angew. Math.), 94 (1883), pp. 41-73.

23. W. Jordan, O. Eggert, and M. Kneissl, Feld- und Landmessung, Handbuch der Vermessungskunde, Metzler, Stuttgart, 1888.

24. S. Kangshen, J. N. Crossley, and A. W.-C. Lun, The Nine Chapters on the Mathematical Art, Companion and Commentary, Oxford University Press, Oxford, 2000 (English transl. from Chinese).

25. G. Kauermann, On the analysis of independencies in margins of a multivariate distribution, Dissertation, Department of Economics, Technical University Berlin, 1994.

26. G. Kauermann, On a dualization of graphical Gaussian models, Scand. J. Stat., 23 (1996), pp. 105-116.

27. S. L. Lauritzen, Graphical Models, Oxford University Press, Oxford, 1996.

28. S. L. Lauritzen and N. Wermuth, Graphical models for associations between variables, some of which are qualitative and some quantitative, Ann. Stat., 17 (1989), pp. 31-54.

29. H. G. Leimer, Optimal decomposition by clique separators, Discrete Math., 113 (1993), pp. 99-123.

30. G. M. Marchetti, Independencies induced from a graphical Markov model after marginalization and conditioning: the $R$ package ggm, J. Stat. Softw., 6 (2006), pp. 1-15.

31. A. A. Markov, Wahrscheinlichkeitsrechnung, Teubner, Leipzig, 1912 (German transl. of 2nd Russian edn. 1908).

32. C. G. Neumann, Vorlesungen über Riemann'sche Theorie der Abel'schen Integrale, 2nd edn., Teubner, Leipzig, 1884.

33. J. Pearl, Probabilistic Reasoning in Intelligent Systems, Morgan Kaufman, San Mateo, 1988.

34. B. W. Peyton, A. Pothen, and X. Yuan, Partitioning a chordal graph into transitive subgraphs for parallel sparse triangular solution, Linear Algebra Appl., 192 (1993), pp. 329354.

35. T. S. Richardson, Markov properties for acyclic directed mixed graphs, Scand. J. Stat., 30 (2003), pp. 145-157.

36. E. Schmidt, Entwicklungen willkürlicher Funktionen, Math. Ann., 63 (1907), pp. 433-476. 
37. J. Q. Smith, Influence diagrams for statistical modelling, Ann. Stat., 17 (1989), pp. 654672.

38. M. Studený and R. Bouckaert, On chain graphs models for description of conditional independence structures, Ann. Stat., 26 (1998), pp. 1434-1495.

39. A. M. Turing, Rounding-off errors in matrix processes, Quart. J. Mech. Appl. Math, 1 (1948), pp. 287-308.

40. K. Vogel, Neun Bücher arithmetischer Technik, Viehweg, Braunschweig, 1968 (German transl. from Chinese).

41. N. Wermuth, On block-recursive linear regression equations (with discussion), Braz. J. Probab. Stat. (REBRAPE, Revista Braslileira de Probilidade e Estatistica), 6 (1992), pp. 1-56.

42. N. Wermuth, Graphical chain models, in Encyclopedia of Behavioral Statistics II, B. Everitt and D. C. Howell, eds., Wiley, Chichester, 2005, pp. 755-757.

43. N. Wermuth and D. R. Cox, On association models defined over independence graphs, Bernoulli, 4 (1998), pp. 477-494.

44. N. Wermuth and D. R. Cox, Joint response graphs and separation induced by triangular systems, J. R. Stat. Soc., Ser. B, 66 (2004), pp. 687-717.

45. H. O. Wold, Causality and econometrics, Econometrica, 22 (1954), pp. 162-177.

46. S. Wright, The theory of path coefficients: a reply to Niles' Criticism, Genetics, 8 (1923), pp. 239-255.

47. S. Wright, The method of path coefficients, Ann. Math. Stat., 5 (1934), pp. 161-215. 